\title{
Researchers sound alarm on 'silent' drug interactions
}

The number of people concurrently taking multiple prescription drugs is on the rise. According to numbers released last fall by the US Centers for Disease Control and Prevention, one in ten Americans is now on five or more medications-nearly twice as many people as in 2000 - and the number of people taking at least two drugs has risen from a quarter to a third of the population over the same time period. Similar statistics are also reported throughout Europe.

And as drug combinations grow more common, so does the risk that these medicines may interact in unpredictable ways that may go undetected until it's too late. Indeed, researchers across the world say that such 'silent' drug interactions pose a growing and widely misunderstood threat.

"Clear drug interactions, something as obvious as a cardiovascular problem or a rash, are easy to weed out and are usually clearly defined before a drug ever comes to market," says Erik Eliasson of the Karolinska University Hospital Huddinge in Stockholm.

"But interactions that don't have immediate effects can take years to understand."

According to Eliasson, many of these interactions take the form of inhibiting the metabolism of drugs that must first be activated by the body to have an effect. One of the most famous examples of such interactions involves selective serotonin reuptake inhibitors (SSRIs), which, as a side effect, inhibit the body's conversion of codeine to morphine. This inhibition can block the painkiller's analgesic effect and cause a dangerous buildup of codeine in people on antidepressants.

However, a recent study of the Swedish Prescribed Drug Register revealed that codeine is seemingly still prescribed by doctors without regard to whether or not the patient is taking an SSRI (J. Intern. Med. 268, 540-548, 2010). The study, co-authored by Eliasson, calls for greater attention to this problem.

An even more alarming and common interaction, Eliasson says, is that of SSRIs with the breast cancer drug tamoxifen. The latter drug is partially responsible for decreasing breast cancer mortality by a third and cutting recurrence in half since the late 1970 s. But, beginning in the 1990s, doctors began prescribing certain SSRIs to patients with breast cancer to treat depression as well as the side effects of tamoxifen, such as blushing and rashes.

Previous work has shown that the drop in side effects is actually due to a blocking of tamoxifen's action (J. Natl. Cancer Inst.

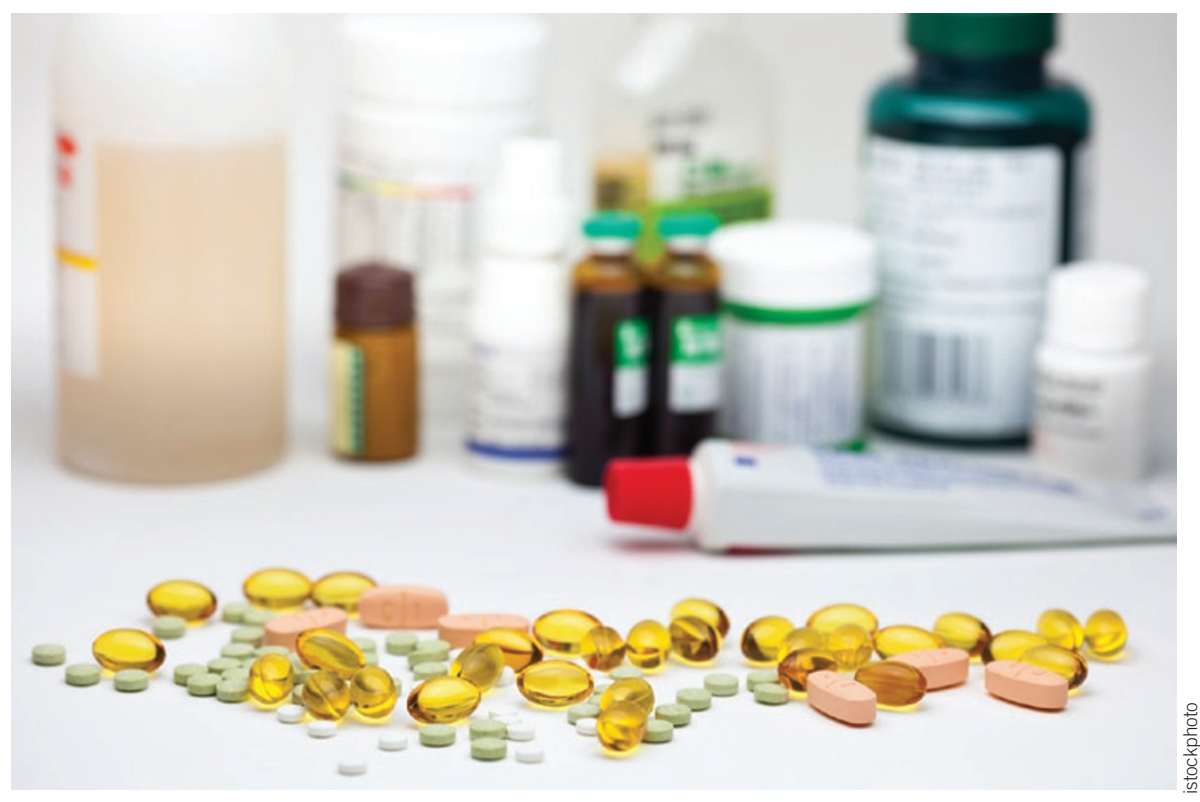

Dangerous synergy: Researchers try to crack down on unexpected drug interactions.

97, 30-39, 2005). And a study published last February of women receiving breast cancer treatment in Ontario between 1993 and 2005 found that women who took certain SSRIs for at least half the time they received tamoxifen were $50 \%$ more likely to die from breast cancer than those only on the latter drug (BMJ 340, c693, 2010). Both the US Food and Drug Administration and the European Medicines Agency are currently considering regulatory action to ban the co-prescribing of these drugs.

Similar interactions can be seen with antiplatelet drugs such as warfarin and hypertension drugs such as losartan.

\section{Prescribing a solution}

One solution to spotting silent interactions is increased physician awareness, says Neil Sandson, a physician with the US Department of Veterans Affairs in Baltimore who has studied drug interactions for nearly two decades.

"Drug companies are becoming more vigilant about searching these interactions out, but some are so subtle that they'll only become apparent when they are actually widely used-and that leaves it up to the doctors who actually doing the prescribing," he says.

Sanderson adds that the implementation of better software to track drug use, as part of the effort to move to electronic medical records, will be vital to screening for silent interactions.
But these tools may not be available in developing nations, where recent improvements to distribution systems for medicine are increasing the use of drug cocktails to fight endemic diseases and therefore increasing the risk of silent drug interactions.

Combinations of drugs to treat illnesses such as HIV, tuberculosis, malaria and hepatitis $\mathrm{C}$ can often interfere with one another, according to David Back and Khoo Saye, researchers at the University of Liverpool in the UK. Certain drugs continuously damage the liver and its production of enzymes that metabolize HIV drugs into their active form. The leads to a gradual drop in the level of the active HIV drugs over the course of months, potentially leading to viral rebound or resistance. And it's often hard to decipher exactly which drug is to blame.

In an as-yet unpublished study, the Liverpool team found that, of 1,000 patients undergoing antiretroviral therapy in Kenya, roughly a quarter had drug interactions that interfered with the HIV treatment-resulting largely from tuberculosis drugs.

"If we think that medical records and tracking tools are important for avoiding interactions in Europe or America, they are possibly even more important in places like Kenya that face widespread devastating diseases with relatively few resources," Back says.

Stu Hutson 